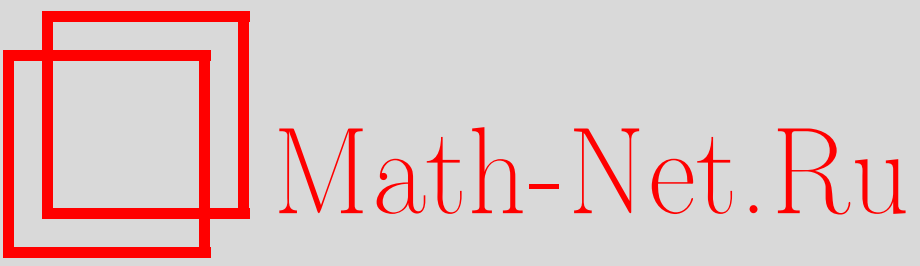

Ш. К. Форманов, О методе Стейна-Тихомирова и его приложениях в неклассических предельных теоремах, Дискрет. матем., 2007, том 19, выпуск 1, 27-39

DOI: https://doi.org/10.4213/dm5

Использование Общероссийского математического портала Math-Net.Ru подразумевает, что вы прочитали и согласны с пользовательским соглашением

http://www . mathnet.ru/rus/agreement

Параметры загрузки:

IP : 107.22 .136 .117

26 апреля 2023 г., 17:38:43 


\title{
Аискретная математика
}

том 19 выпуск $1 * 2007$

УДК 519.2

\author{
О методе Стейна-Тихомирова \\ и его приложениях в неклассических \\ предельных теоремах
}

() 2007 г. Ш. К. Форманов

\begin{abstract}
В работе приведены необходимые и достаточные условия справедливости центральной предельной теоремы для сумм независимых случайных величин в терминах характеристических функций в неклассической постановке, когда допускается существенный вклад отдельных слагаемых в сумму. Эти условия сформулированы с использованием введенного в статье оператора Стейна-Тихомирова.

Статья написана на основе доклада, прочитанного на VI международной Петрозаводской конференции “Вероятностные методы в дискретной математике”, состоявшейся в Петрозаводске в 2004 году.
\end{abstract}

\section{1. Модифицированный вариант метода Стейна-Тихомирова}

В теории суммирования независимых и слабо зависимых случайных величин все большую популярность приобретает метод Стейна, основы которого изложены, например, в $[1,2]$. Суть этого метода заключается в следующем: пусть требуется доказать предельную теорему о том, что последовательность функций распределений $\left\{F_{n}, n \geqslant 1\right\}$ слабо сходится к функции распределения $F(x)$. Для этого предварительно изучается задача о характеризации распределения путем аннулирования некоторого соотношения для функции распределения $F(x)$. После этого доказывается, что это утверждение имеет место для функции распределения $F_{n}$ в асимптотическом смысле (при больших $n$ ). Например, в случае центральной предельной теоремы важную роль играет следующее предложение.

Лемма 1 ([2]). Пусть $F(x)-$ функиия распределения, для которой

$$
\int_{-\infty}^{\infty} x d F(x)=0, \quad \int_{-\infty}^{\infty} x^{2} d F(x)=1 .
$$

Тогда для того чтобь

$$
F(x)=\Phi(x)=\frac{1}{\sqrt{2 \pi}} \int_{-\infty}^{x} e^{-u^{2} / 2} d u,
$$

необходимо и достаточно выполнения равенства

$$
\int_{-\infty}^{\infty} g^{\prime}(x) d F(x)=\int_{-\infty}^{\infty} x g(x) d F(x)
$$


для любой кусочно непрерывно дифферениируемой функиии g такой, что

$$
\int_{-\infty}^{\infty}\left|g^{\prime}(x)\right| e^{-x^{2} / 2} d x<\infty
$$

Применение метода Стейна сопряжено со следующим дифференциальным уравнением (см. [1, 2]):

$$
y^{\prime}(u)-u y(u)=h(u)-N h,
$$

где $h(u)$ - ограниченная борелевская функция на прямой и функционал

$$
N h=\frac{1}{\sqrt{2 \pi}} \int_{-\infty}^{\infty} h(x) e^{-x^{2} / 2} d x .
$$

Для конкретных применений метода Стейна следует решить уравнение (2) в классе кусочно непрерывно дифференцируемых функций $g$, для которых выполняется условие (1). В первоначальной работе Ч. Стейна [1] в качестве функции $h$ рассмотрена индикаторная функция

$$
h(u)=h_{x}(u)=I_{(-\infty, x)}(u), \quad x \in \mathbf{R} .
$$

В этом случае непосредственно можно убедиться в том, что

$$
y(u)=y_{x}(u)= \begin{cases}\sqrt{2 \pi} e^{u^{2} / 2} \Phi(u)(1-\Phi(x)) & \text { при } u \leqslant x, \\ \sqrt{2 \pi} e^{u^{2} / 2}(1-\Phi(u)) \Phi(x) & \text { при } u>x .\end{cases}
$$

Пусть $\xi-$ произвольная случайная величина с функцией распределения $F(x)$. Положив в (2) $u=\xi$ и взяв математическое ожидание от обеих частей этого равенства, получаем, что

$$
F(x)-\Phi(x)=\mathbf{E} y_{x}^{\prime}(\xi)-\mathbf{E} \xi y_{x}(\xi)
$$

(при этом следует учитывать равенство $\Phi(x)=N h_{x}$ ).

Таким образом, оценка отклонения $F(x)$ от $\Phi(x)$ сводится к оценке величины (характеристики нормальности)

$$
\sup _{x}\left|\mathbf{E} y_{x}^{\prime}(\xi)-\mathbf{E} \xi y_{x}(\xi)\right|,
$$

где $y_{x}$ определяется равенством (3). Последнее согласуется с леммой 1 , и при этом можно убедиться в том, что $N y_{x}<\infty$ для любого $x \in \mathbf{R}$ (см. лемму 2 в [2]). Из сказанного и леммы 1 получаем удобный вариант доказательства центральной предельной теоремы: если дана последовательность случайных величин $w_{1}, w_{2}, \ldots, w_{n}, \ldots$, для которых выполняется равенство

$$
\lim _{n \rightarrow \infty} \mathbf{E}\left|g^{\prime}\left(w_{n}\right)-w_{n} g\left(w_{n}\right)\right|=0,
$$

то распределение случайных величин $w_{n}$ асимптотически нормально.

В цитированной работе [1] Ч. Стейн высказал мнение о том, что его метод не имеет никакого отношения к известному методу характеристических функций. Но А. Н. Тихомиров [3] на примере оценок скорости сходимости в центральной предельной теореме показал, что именно сочетание методов Стейна и характеристических функций может играть важную роль в теории суммирования слабо зависимых случайных величин. В последующем 
приемы доказательств центральной предельной теоремы и оценки остаточного члена в этой теореме, использующие идеи работ $[1,3]$, в собирательном смысле стали называться методом Стейна-Тихомирова. В работе [4] автора был предложен модифицированный (по отношению к ранее используемым) вариант метода Стейна-Тихомирова, который основывается на следующем характеризационном свойстве нормального распределения в терминах характеристических функций.

Лемма 2. Для того чтобы случайная величина $w$ c $\mathbf{E} w=0$, $\mathbf{E} w^{2}=\sigma^{2}$ имела нормальное распределение с параметрами $\left(0, \sigma^{2}\right)$ необходимо и достаточно, чтобы ее характеристическая функция удовлетворяла равенству

$$
f^{\prime}(t)=-\sigma^{2} t f(t) .
$$

Если рассмотреть равенство (6) как дифференциальное уравнение с начальным условием $f(0)=1$, то утверждение леммы 2 становится очевидным.

Теперь, основываясь на равенстве (6), в классе характеристических функций

$$
\mathbf{F}_{N}=\left\{f(t): f^{\prime}(0)=0, f^{\prime \prime}(0)=-\sigma^{2}>-\infty\right\}
$$

введем оператор Стейна-Тихомирова

$$
\Delta(f(t))=f^{\prime}(t)+\sigma^{2} t f(t) .
$$

Последнее равенство (7) представляет собой линейное дифференциальное уравнение с правой частью $\Delta(f(t))$ и с начальным условием $f(0)=1$. Из его решения (см., например, стр. 28 в [5]) получаем, что

$$
f(t)-e^{-\sigma^{2} t^{2} / 2}=e^{-\sigma^{2} t^{2} / 2} \int_{0}^{t} \Delta(f(s)) e^{\sigma^{2} s^{2} / 2} d s .
$$

Здесь знаки переменной интегрирования $s$ и величины $t$ совпадают, причем $|s| \leqslant|t|$. Из равенств (6) и (8) также следует, что равенство

$$
\Delta(f(t))=0
$$

полностью характеризует характеристическую функцию нормального распределения с параметрами $\left(0, \sigma^{2}\right)$. В свою очередь, из леммы 2 и равенства (9) вытекает, что если для любого $t \in \mathbf{R}$

$$
\lim _{n \rightarrow \infty} \Delta\left(\mathbf{E} e^{i t w_{n}}\right)=0
$$

то распределение случайной величины $w_{n}$ асимптотически нормально.

Метод доказательства центральной предельной теоремы, основанный на предельном соотношении (10), может быть рассмотрен как модифицированный вариант метода Стейна-Тихомирова, и во многих случаях проверка выполнения равенства (10) проще по сравнению с проверкой условия (5).

В приложениях к проблемам теории суммирования независимых случайных величин важную роль играет следующее свойство оператора $\Delta$.

Лемма 3. Пусть характеристические функции $f, g \in \mathbf{F}_{N}$. Тогда

$$
\Delta(f g)=f \Delta(g)+g \Delta(f) .
$$

то есть оператор по отношению к произведению характеристических функций представляет собой операцию дифференцирования. 
Доказательство. Пусть характеристические функции $f, g \in \mathbf{F}_{N}$ и

$$
\sigma_{1}^{2}=-f^{\prime \prime}(0), \quad \sigma_{2}^{2}=-g^{\prime \prime}(0) .
$$

Тогда по определению оператора $\Delta$

$$
\begin{aligned}
\Delta(f g) & =(f g)^{\prime}+t\left(-\left.(f g)^{\prime \prime}\right|_{t=0}\right) f g=f^{\prime} g+g^{\prime} f+t\left(\sigma_{1}^{2}+\sigma_{2}^{2}\right) f g \\
& =\left(f^{\prime}+\sigma_{1}^{2} t f\right) g+f\left(g^{\prime}+\sigma_{2}^{2} t g\right)=f \Delta(g)+g \Delta(f) .
\end{aligned}
$$

Лемма 3 доказана.

\section{2. Неклассические варианты центральной предельной теоремы в терминах характеристических функций}

Основным объектом исследования теории суммирования независимых случайных величин, называемой также центральной предельной проблемой, являются последовательности сумм

$$
S_{n}=X_{n 1}+X_{n 2}+\ldots+X_{n k_{n}},
$$

где $X_{n 1}, X_{n 2}, \ldots$ - последовательность независимых случайных величин, образующих схему серий (число слагаемых $k_{n}$ может быть как конечным, так и бесконечным). При этом основной задачей является описание класса законов распределений, которые могут выступать в качестве предельных (в смысле слабой сходимости) для распределения суммы $S_{n}$. На этом пути и появляется ограничение, известное под названием условия равномерной бесконечной малости: при $n \rightarrow \infty$

$$
\sup _{j} \mathbf{P}\left(\left|X_{n j}\right| \geqslant \varepsilon\right) \rightarrow 0
$$

при любом $\varepsilon>0$.

Необходимость появления условия (12) объясняется тем, что при его выполнении предельный закон становится нечувствительным к поведению отдельных слагаемых. Но при решении задач, связанных с отысканием условий сходимости распределения суммы $S_{n}$ к заданному закону, введение ограничения типа условия равномерной бесконечной малости (12) не представляется обязательным (см. [6]). В качестве тривиального примера сходимости к заданному невырожденному распределению с нарушением условия (12) можно указать последовательность

$$
\mathbf{P}\left(X_{n 1}<x\right)=F_{n 1}=F(x), \quad X_{n j}=0, \quad j \geqslant 2 .
$$

Следуя В. М. Золотареву [6], предельные теоремы для распределения сумм $S_{n}$, доказанные без привлечения условия (12), называют неклассическими.

Далее будем предполагать, что

$$
\mathbf{E} X_{n j}=0, \quad \sigma_{n j}^{2}=\mathbf{D} X_{n j}, \quad j=1,2, \ldots, \quad \sum_{j} \sigma_{n j}^{2}=1 .
$$

В случае выполнения (13) условие (12) превращается в следующее условие: при $n \rightarrow \infty$

$$
\max _{j} \sigma_{n j}^{2} \rightarrow 0
$$


Как хорошо известно, в предельных теоремах теории вероятностей важную роль играет расстояние Леви, которое для функций распределения $F$ и $G$ определяется формулой

$$
L(F, G)=\inf \{\varepsilon>0, G(x-\varepsilon)-\varepsilon \leqslant F(x) \leqslant G(x+\varepsilon)+\varepsilon,-\infty<x<\infty\} .
$$

Особое значение этого расстояния заключается в том, что оно метризует слабую сходимость распределений. Дополнительно к введенным обозначениям положим

$$
F_{n}(x)=\mathbf{P}\left(S_{n}<x\right), \quad F_{n j}(x)=\mathbf{P}\left(X_{n j}<x\right), \quad \Phi_{n j}(x)=\Phi\left(\frac{x}{\sigma_{n j}}\right), \quad j=1,2, \ldots
$$

В этих обозначениях утверждение центральной предельной теоремы равносильно тому, что при $n \rightarrow \infty$

$$
L\left(F_{n}, \Phi\right) \rightarrow 0
$$

Первым неклассическим вариантом центральной предельной теоремы является следующая теорема, доказанная В. М. Золотаревым [6].

Теорема А. Сходимость (15) имеет место тогда и только тогда, когда при неограниченном возрастании п оказываются выполненными следующие два условия:

$$
\alpha_{n}=\sup _{j} L\left(F_{n j}, \Phi_{n j}\right) \rightarrow 0
$$

при каждом положительном $\varepsilon$

$$
L_{n}(\varepsilon)=\sum_{j \in A_{n}} \int_{|x| \geqslant \varepsilon} x^{2} d F_{n j} \rightarrow 0
$$

где множество $A_{n}$ содержит те значения индексов $j$, для которых

$$
\sigma_{n j}^{2} \leqslant \sqrt{\alpha_{n}}
$$

Смысл условий (16)-(18) сводится к тому, что сначала в сумме $S_{n}$ выделяются те слагаемые, которые удовлетворяют условию равномерной бесконечной малости (14), а потом для этих слагаемых требуется выполнение классического условия Линдеберга. С другой стороны, легко видеть, что если выполнено условие (14), то выполняется условие (16) $\left(\alpha_{n} \rightarrow 0\right)$. Таким образом, теорема А представляет собой обобщение классической теоремы Линдеберга-Феллера.

В работе В. И. Ротаря [7] условия (16), (17) теоремы А заменяются одним другим условием.

Теорема В. Пусть выполнено условие (13). При $n \rightarrow \infty$

$$
\sup _{x}\left|F_{n}(x)-\Phi(x)\right| \rightarrow 0
$$

тогда и только тогда, когда при любом $\varepsilon>0$ при $n \rightarrow \infty$

$$
R_{n}(\varepsilon)=\sum_{j} \int_{|x| \geqslant \varepsilon}|x|\left|F_{n j}(x)-\Phi_{n j}(x)\right| d x \rightarrow 0 .
$$


Поскольку функция нормального распределения $\Phi(x)$ является непрерывной в любой точке, утверждение $L\left(F_{n}, \Phi\right) \rightarrow 0$ равносильно (19). Далее, условие (20) никак не связано с условием равномерной бесконечной малости (14), и поэтому теорема В является неклассическим вариантом центральной предельной теоремы.

Представляют особый интерес задачи о нахождении необходимых и достаточных условий для справедливости центральной предельной теоремы в неклассической ситуации в терминах характеристических функций. Следующие соображения могут служить обоснованием правомерности использования условий на характеристические функции в задачах, связанных с центральной предельной теоремой. Во-первых, характеристическая функция однозначно определяет распределение соответствующей случайной величины. Во-вторых, условия в терминах характеристических функций могут иметь более простой вид по сравнению с условиями, формулируемыми в терминах функций распределения.

Теорема 1. При условии (13)

$$
\sup _{x}\left|F_{n}(x)-\Phi(x)\right| \rightarrow 0
$$

при $n \rightarrow \infty$ тогда и только тогда, когда при любом $t$

$$
\lim _{n \rightarrow \infty} \sum_{j} \mid \Delta\left(f_{n j}(t) \mid=0\right.
$$

где $f_{n j}(t)=\mathbf{E} e^{i t X_{n j}}-$ характеристическая функция случайной величины $X_{n j}$.

Доказательство. Доказательство достаточности совсем простое, в особенности, по сравнению с приводимыми во многих книгах доказательствами теоремы Линдеберга (см., например, §4 гл. 8 в [10]). Действительно, если положить

$$
f_{n}(t)=\mathbf{E} e^{i t S_{n}}
$$

то для доказательства (19), согласно (10), достаточно убедиться в том, что

$$
\lim _{n \rightarrow \infty}\left|\Delta\left(f_{n}(t)\right)\right|=0
$$

В свою очередь, в силу леммы 3,

$$
\Delta\left(f_{n}(t)\right)=\sum_{j} \prod_{k \leqslant j-1} f_{n k}(t) \Delta\left(f_{n j}(t)\right) \prod_{k \geqslant j+1} f_{n k}(t) .
$$

Следовательно,

$$
\left|\Delta\left(f_{n}(t)\right)\right| \leqslant \sum_{j}\left|\Delta\left(f_{n j}(t)\right)\right| .
$$

Теперь соотношения (21)-(23) доказывают достаточность условия теоремы 1.

Докажем необходимость условия (21).

Воспользовавшись равенством

$$
\Delta\left(\varphi_{n j}(t)\right)=\Delta\left(e^{-\sigma_{n j}^{2} t^{2} / 2}\right)=0,
$$


справедливым при любом $j$, находим, что

$$
\begin{aligned}
\sum_{j}\left|\Delta\left(f_{n j}(t)\right)\right| & =\sum_{j} \mid \Delta\left(f_{n j}(t)\right)-\Delta\left(\varphi_{n j}(t) \mid\right. \\
& =\sum_{j}\left|\left(f_{n j}^{\prime}(t)-\varphi_{n j}^{\prime}(t)\right)+t \sigma_{n j}^{2}\left(f_{n j}(t)-\varphi_{n j}(t)\right)\right| \leqslant \Sigma_{n 1}(t)+\Sigma_{n 2}(t),
\end{aligned}
$$

где

$$
\begin{aligned}
& \Sigma_{n 1}=\sum_{j}\left|f_{n j}^{\prime}(t)-\varphi_{n j}^{\prime}(t)\right|, \\
& \Sigma_{n 2}=|t| \sum_{j} \sigma_{n j}^{2}\left|f_{n j}(t)-\varphi_{n j}(t)\right| .
\end{aligned}
$$

Оценим сначала $\Sigma_{n 2}(t)$. С учетом того, что

$$
\mathbf{E} X_{n j}=0, \quad \sigma_{n j}^{2}=\int_{-\infty}^{\infty} x^{2} d F_{n j}=\int_{-\infty}^{\infty} x^{2} d \Phi_{n j},
$$

проведя интегрирование по частям, получаем, что

$$
\begin{aligned}
\left|f_{n j}(t)-\varphi_{n j}(t)\right| & =\left|\int_{-\infty}^{\infty} e^{i t x} d\left(F_{n j}-\Phi_{n j}\right)\right| \\
& =\left|\int_{-\infty}^{\infty}\left(e^{i t x}-1-i t x+\frac{(i t x)^{2}}{2}\right) d\left(F_{n j}-\Phi_{n j}\right)\right| \\
& =\left|\int_{-\infty}^{\infty} i t\left(e^{i t x}-1-i t x\right)\left(F_{n j}-\Phi_{n j}\right) d x\right| \leqslant I_{1}+I_{2},
\end{aligned}
$$

где

$$
\begin{aligned}
& I_{1}=|t| \int_{|x| \leqslant \varepsilon}\left|e^{i t x}-1-i t x\right|\left|F_{n j}-\Phi_{n j}\right| d x, \\
& I_{2}=|t| \int_{|x|>\varepsilon}\left|e^{i t x}-1-i t x\right|\left|F_{n j}-\Phi_{n j}\right| d x .
\end{aligned}
$$

Воспользовавшись неравенством

$$
\left|e^{i t x}-1-i t x\right| \leqslant \frac{t^{2} x^{2}}{2}
$$

находим, что

$$
\begin{aligned}
I_{1} & \leqslant \frac{|t|^{3}}{2} \int_{|x| \leqslant \varepsilon}|x|^{2}\left|F_{n j}-\Phi_{n j}\right| d x \leqslant \frac{|t|^{3}}{2} \varepsilon \int_{|x| \leqslant \varepsilon}|x|\left|F_{n j}-\Phi_{n j}\right| d x \\
& =\frac{|t|^{3}}{2} \varepsilon\left(\int_{-\varepsilon}^{0}(-x)\left|F_{n j}-\Phi_{n j}\right| d x+\int_{0}^{\varepsilon} x\left|F_{n j}-\Phi_{n j}\right| d x\right) \\
& \leqslant \frac{|t|^{3}}{2} \varepsilon\left(\int_{0}^{\varepsilon} x\left(1-F_{n j}(x)+F_{n j}(-x)\right) d x+\int_{0}^{\varepsilon} x\left(1-\Phi_{n j}(x)+\Phi_{n j}(-x)\right) d x\right) .
\end{aligned}
$$

2 Дискретная математика, т.19 №1 
Непосредственно можно убедиться в том, что для функции распределения $F(x)$

$$
\int_{\infty}^{\infty} x^{2} d F(x)=2 \int_{0}^{\infty} x(1-F(x)+F(-x)) d x .
$$

Теперь из (26), (27) получаем, что

$$
I_{1} \leqslant \frac{|t|^{3}}{4} \varepsilon\left(\sigma_{n j}^{2}+\sigma_{n j}^{2}\right)=\frac{|t|^{3}}{2} \varepsilon \sigma_{n j}^{2} .
$$

Далее, воспользовавшись неравенством

$$
\left|e^{i \alpha}-1\right| \leqslant|\alpha|, \quad \alpha \in \mathbf{R}
$$

находим, что

$$
I_{2} \leqslant 2|t|^{2} \int_{|x|>\varepsilon}|x|\left|F_{n j}(x)-\Phi_{n j}(x)\right| d x .
$$

Проведя суммирование по $j$ в оценках (28) и (29), с учетом (25) получаем, что

$$
\begin{aligned}
\sum_{j}\left|f_{n j}(t)-\varphi_{n j}(t)\right| & \leqslant \frac{|t|^{3}}{2} \varepsilon+2 t^{2} \int_{|x|>\varepsilon}|x|\left|F_{n j}(x)-\Phi_{n j}(x)\right| d x \\
& =\frac{|t|^{3}}{2} \varepsilon+2 t^{2} R_{n}(\varepsilon) .
\end{aligned}
$$

Из теоремы В (см. (20)) и последнего соотношения (30), в силу произвольности $\varepsilon>0$, следует, что при любом $t$

$$
\lim _{n \rightarrow \infty} \Sigma_{n 2}(t)=0
$$

Займемся теперь оценкой $\Sigma_{n 1}(t)$. Предварительно заметим, что

$$
\left|f_{n j}^{\prime}(t)-\varphi_{n j}^{\prime}(t)\right|=\left|\int_{-\infty}^{\infty} x\left(e^{i t x}-1-i t x\right) d\left(F_{n j}-\Phi_{n j}\right)\right| .
$$

Произведя интегрирование по частям в интеграле под знаком модуля, получаем, что

$$
\left|f_{n j}^{\prime}(t)-\varphi_{n j}^{\prime}(t)\right| \leqslant I_{3}+I_{4}
$$

где

$$
\begin{aligned}
& I_{3}=\left|\int_{-\infty}^{\infty}\left(e^{i t x}-1-i t x\right)\left(F_{n j}-\Phi_{n j}\right) d x\right|, \\
& I_{4}=|t|\left|\int_{-\infty}^{\infty} x\left(e^{i t x}-1\right)\left(F_{n j}-\Phi_{n j}\right) d x\right| .
\end{aligned}
$$

Очевидно, что

$$
\begin{aligned}
I_{3} \leqslant & \frac{t^{2}}{2} \int_{|x| \leqslant \varepsilon} x^{2}\left|F_{n j}-\Phi_{n j}\right| d x+\int_{|x|>\varepsilon}\left|e^{i t x}-1\right|\left|F_{n j}-\Phi_{n j}\right| d x \\
& +|t| \int_{|x|>\varepsilon}|x|\left|F_{n j}-\Phi_{n j}\right| d x \\
& \leqslant \frac{t^{2}}{2} \varepsilon \int_{|x| \leqslant \varepsilon}|x|\left|F_{n j}-\Phi_{n j}\right| d x+2|t| \int_{|x|>\varepsilon}|x|\left|F_{n j}-\Phi_{n j}\right| d x .
\end{aligned}
$$


Из равенства (27) получаем, что

$$
\int_{|x| \leqslant \varepsilon}|x|\left|F_{n j}-\Phi_{n j}\right| d x \leqslant 2 \sigma_{n j}^{2} .
$$

С учетом соотношений (32)-(34) можно получить оценку

$$
I_{3} \leqslant t^{2} \varepsilon \sigma_{n j}^{2}+2|t| \int_{|x|>\varepsilon}|x|\left|F_{n j}-\Phi_{n j}\right| d x .
$$

Далее, имея ввиду неравенство $\left|e^{i t x}-1\right| \leqslant 2$, получаем оценку

$$
I_{4} \leqslant t^{2} \varepsilon \int_{|x| \leqslant \varepsilon}|x|\left|F_{n j}-\Phi_{n j}\right| d x+2|t| \int_{|x|>\varepsilon}|x|\left|F_{n j}-\Phi_{n j}\right| d x .
$$

В свою очередь, принимая во внимание (34), находим, что

$$
I_{4} \leqslant 2 t^{2} \varepsilon \sigma_{n j}^{2}+2|t| \int_{|x|>\varepsilon}|x|\left|F_{n j}-\Phi_{n j}\right| d x .
$$

Из соотношений (32), (35), (36) окончательно получаем, что

$$
\sum_{j}\left|f_{n j}^{\prime}(t)-\varphi_{n j}^{\prime}(t)\right| \leqslant \frac{5}{2} t^{2} \varepsilon+4|t| R_{n}(\varepsilon) .
$$

В силу произвольности $\varepsilon>0$ из (37) следует, что при любом $t$

$$
\lim _{n \rightarrow \infty} \Sigma_{n 1}(t)=0
$$

Необходимость условия (21) следует из соотношений (24), (31), (38). Теорема 1 доказана.

Замечание 1. Поскольку характеристические функции $f_{n j}(t)$ и $f_{n j}^{\prime}(t)$ являются равномерно непрерывными на любом конечном отрезке, условие (21) теоремы 1 может быть записано в виде

$$
\lim _{n \rightarrow \infty} \sup _{|t| \leqslant T} \sum_{j}\left|\Delta\left(f_{n j}(t)\right)\right|=0
$$

при любом $T>0$.

Замечание 2. Как видно из доказательства теоремы 1, по части необходимости в нем существенную роль играет введенная Ротарем характеристика $R_{n}(\varepsilon)$ из теоремы В. Фактически здесь доказана справедливость импликации

$$
\left\{R_{n}(\varepsilon) \rightarrow 0\right\} \Longrightarrow(21)
$$

В свою очередь, в работе [7] доказано, что условие (17) не сильнее совместного выполнения условий (16) и (17) из теоремы А. Таким образом, теоремы А и В, а также теорема 1 являются эквивалентными вариантами неклассической центральной предельной теоремы, обобщающими классическую теорему Линдеберга-Феллера.

Следующая теорема является аналогом теоремы Золотарева в терминах характеристических функций. 
Теорема 2. Для того чтобы при $n \rightarrow \infty$ имела место сходимость

$$
L\left(F_{n}, \Phi\right) \rightarrow 0
$$

необходимо и достаточно выполнения следующих условий:

(1) $\alpha_{n} \rightarrow 0$ при $n \rightarrow \infty$,

(2) для любого $t \in \mathbf{R}$ при $n \rightarrow \infty$

$$
\sum_{j \in A_{n}}\left|\Delta\left(f_{n j}(t)\right)\right| \rightarrow 0,
$$

где множество индексов $A_{n}$ определяется равенством (18).

Достаточность условий 1 и 2 этой теоремы докажем, используя теорему 1 , согласно которой нам достаточно показать, что при любом $t$ при $n \rightarrow \infty$

$$
\sum_{j}\left|\Delta\left(f_{n j}(t)\right)\right| \rightarrow 0
$$

(см. соотношение (21)).

Ясно, что

$$
\sum_{j}\left|\Delta\left(f_{n j}(t)\right)\right|=\Sigma_{n}^{\prime}(t)+\Sigma_{n}^{\prime \prime}(t)
$$

где

$$
\begin{aligned}
& \Sigma_{n}^{\prime}(t)=\sum_{j \in A_{n}}\left|\Delta\left(f_{n j}(t)\right)\right|, \\
& \Sigma_{n}^{\prime \prime}(t)=\sum_{j \in \bar{A}_{n}}\left|\Delta\left(f_{n j}(t)\right)\right| .
\end{aligned}
$$

Согласно условию 2 для любого $t$ при $n \rightarrow \infty$

$$
\Sigma_{n}^{\prime}(t)=o(1)
$$

Учитывая равенства $\Delta\left(\varphi_{n j}(t)\right)=0, j=1,2, \ldots$, находим, что

$$
\Sigma_{n}^{\prime \prime}(t)=\sum_{j \in \bar{A}_{n}}\left|\Delta\left(f_{n j}(t)\right)\right| \leqslant \Sigma_{n 1}^{\prime}(t)+\Sigma_{n 2}^{\prime \prime}(t)
$$

где

$$
\begin{aligned}
& \Sigma_{n 1}^{\prime}(t)=\sum_{j \in \bar{A}_{n}}\left|f_{n j}^{\prime}(t)-\varphi_{n j}^{\prime}(t)\right|, \\
& \Sigma_{n 2}^{\prime \prime}(t)=|t| \sum_{j \in \bar{A}_{n}} \sigma_{n j}^{2}\left|f_{n j}(t)-\varphi_{n j}(t)\right| .
\end{aligned}
$$

Для всех $j \in \bar{A}_{n}$ имеет место неравенство $\sigma_{n j}^{2} \geqslant \sqrt{\alpha_{n}}$, поэтому (см. (13))

$$
\sum_{j \in \bar{A}_{n}} \sigma_{n j}^{2} \geqslant \sqrt{\alpha_{n}} \sum_{j \in \bar{A}_{n}} 1
$$


Следовательно,

$$
\sum_{j \in \bar{A}_{n}} 1 \leqslant \frac{1}{\sqrt{\alpha_{n}}}
$$

Заметим, что в последнее время в области предельных теорем часто используется определяемая для пары функций распределения $F, G$ и называемая $l$-м разностным моментом характеристика (см. [6])

$$
v_{l}(F, G)=l \int_{-\infty}^{\infty}|x|^{l-1}|F(x)-G(x)| d x .
$$

Нетрудно убедиться в том, что

$$
v_{l}(F, G) \leqslant \mathbf{E}|X|^{l}+\mathbf{E}|Y|^{l}
$$

где $X$ и $Y$ - случайные величины, имеющие соответственно функции распределения $F$ и $G$, причем, если при этом $\mathbf{P}(Y=0)=1$, то последнее неравенство можно заменить на равенство. В [8] указано на связь разностного момента с известной метрикой Канторовича-Рубинштейна [9], а именно,

$$
v_{l}(F, G)=\left.\inf \mathbf{E}|X| X\right|^{l-1}-Y|Y|^{l-1} \mid,
$$

где инфимум берется по всем совместным распределениям случайного вектора $(X, Y)$ с маргинальными распределениями $F$ и $G$.

Для любой функции распределения $F(x)$ положим

$$
F^{*}(x)=1-F(x)+F(-x) .
$$

Тогда условие совпадения дисперсий для случайных величин с функциями распределения $F$ и $G$ (имеющих нулевые средние) может быть записано в виде

$$
\sigma^{2}=\int_{0}^{\infty} x F^{*}(x) d x=\int_{0}^{\infty} x G^{*}(x) d x .
$$

В следующей лемме, доказанной в [7], приводится оценка характеристики

$$
v(F, G)=\frac{v_{2}(F, G)}{2}=\int_{-\infty}^{\infty}|x||F(x)-G(x)| d x
$$

через расстояние Леви $L(F, G)$.

Лемма 4. Пусть выполнено равенство (43). Тогда для любого В >0

$$
v(F, G) \leqslant 4\left(\int_{B}^{\infty} x G^{*}(x) d x+B(B+1) L(F, G)\right) .
$$

Заметим, что для любой функции распределения $F(x)$

$$
2 \int_{\varepsilon}^{\infty} x F^{*}(x) d x \leqslant \int_{|x| \geqslant \varepsilon} x^{2} d F(x)
$$


Положив в (44) $\bar{G}(x)=G(x \sigma), B=A \sigma$, с учетом (45) для любого $A \geqslant 1$ получаем, что

$$
v(F, G) \leqslant 4\left(\int_{|x|>A} x^{2} d \bar{G}(x)+A^{2}\left(\sigma^{2}+\sigma\right) L(F, G)\right) .
$$

Теперь применим приведенные рассуждения к оценке $\Sigma_{n 1}^{\prime \prime}(t)$. Предварительно при помощи оценки

$$
\left|e^{i \alpha}-1\right| \leqslant \min (|\alpha|, 2)
$$

из равенства (32) получим, что

$$
\left|f_{n j}^{\prime}(t)-\varphi_{n j}^{\prime}(t)\right| \leqslant 4|t| \int_{-\infty}^{\infty}|x|\left|F_{n j}-\Phi_{n j}\right| d x=4|t| v\left(F_{n j}, \Phi_{n j}\right) .
$$

Воспользовавшись соотношениями (46), (47), получаем следующую цепочку оценок:

$$
\begin{aligned}
\Sigma_{n 1}^{\prime \prime}(t) & \leqslant 4|t| \sum_{j \in \bar{A}_{n}} v\left(F_{n j}, \Phi_{n j}\right) \leqslant 16|t|\left(\sum_{j \in \bar{A}_{n}} \sigma_{n j}^{2} \int_{|x|>A} x^{2} d \Phi(x)+A^{2} \sum_{j \in \bar{A}_{n}}\left(\sigma_{n j}^{2}+\sigma_{n j}\right) \alpha_{n}\right) \\
& \leqslant 16|t| \int_{|x|>A} x^{2} d \Phi(x)+16|t| A^{2} \sum_{j \in \bar{A}_{n}}\left(\sigma_{n j}^{2}+\alpha_{n}^{-1 / 4} \sigma_{n j}^{2}\right) \alpha_{n} \\
& \leqslant 16|t| \int_{|x|>A} x^{2} d \Phi(x)+16|t| A^{2}\left(\alpha_{n}+\alpha_{n}^{3 / 4}\right)
\end{aligned}
$$

для любого $A>1$.

При выводе последней оценки в (48) учтено неравенство (42) и то, что для $j \in \overline{A_{n}}$

$$
\sigma_{n j} \leqslant \alpha_{n}^{-1 / 4} \sigma_{n j}^{2} .
$$

Выбирая $A$ достаточно большим и учитывая условия 1 , из (48) получаем, что при $n \rightarrow \infty$ для любого $t$

$$
\Sigma_{n 1}^{\prime \prime}(t)=o(1)
$$

Оценим теперь $\Sigma_{n 2}^{\prime \prime}(t)$. Из соотношений $(25),(28),(29)$ получаем, что

$$
\begin{aligned}
\Sigma_{n 2}^{\prime \prime}(t) & \leqslant|t| \sum_{j \in \bar{A}_{n}} \sigma_{n j}^{2}\left|f_{n j}(t)-\varphi_{n j}(t)\right| \leqslant|t|^{4} \varepsilon \sum_{j \in \bar{A}_{n}} \sigma_{n j}^{2}+2|t|^{3} \sum_{j \in \bar{A}_{n}} \int_{|x|>\varepsilon}|x|\left|F_{n j}-\Phi_{n j}\right| d x \\
& \leqslant|t|^{4} \varepsilon+2|t|^{3} \sum_{j \in \bar{A}_{n}} v\left(F_{n j}, \Phi_{n j}\right) .
\end{aligned}
$$

Последнее слагаемое в (50) оценено в соотношении (48), согласно которому при $n \rightarrow \infty$

$$
\sum_{j \in \bar{A}_{n}} v\left(F_{n j}, \Phi_{n j}\right)=o(1) .
$$

Следовательно, в силу (50), (51), получаем, что для любого $t$ при $n \rightarrow \infty$

$$
\Sigma_{n 2}^{\prime \prime}(t)=o(1)
$$


Теперь из (41), (49), (52) следует, что

$$
\Sigma_{n}^{\prime \prime}(t)=o(1)
$$

и в силу (39), (40), (53) окончательно получаем, что при $n \rightarrow \infty$

$$
\sum_{j}\left|\Delta\left(f_{n j}(t)\right)\right| \rightarrow 0
$$

при любом $t$. Теперь достаточность условий 1 и 2 теоремы 2 следует из соотношения (54) и теоремы 1.

Доказательство необходимости условия $1\left(\alpha_{n} \rightarrow 0\right)$ получено В. М. Золотаревым (см. [6]), а необходимость условия 2 очевидна в силу теоремы 1.

Замечание 3. Хорошо известно, что условие $\sup _{j} L\left(F_{n j}, \Phi_{n j}\right) \rightarrow 0$ при $n \rightarrow \infty$ равносильно тому, что для любого $t$ при $n \rightarrow \infty$

$$
\sup _{j}\left|f_{n j}(t)-\varphi_{n j}(t)\right| \rightarrow 0 \text {. }
$$

В силу равномерной непрерывности характеристической функции последнее эквивалентно утверждению о том, что при $n \rightarrow \infty$

$$
\sup _{j} \sup _{|t| \leqslant T}\left|f_{n j}(t)-\varphi_{n j}(t)\right| \rightarrow 0
$$

для любого $T>0$. Следовательно, условие 1 в теореме 2 может быть заменено одним из условий (55), (56).

\section{Список литературы}

1. Stein C., A bound for the error in the normal approximation to the distribution of a sum of dependent random variables. In: Proc. 6th Berkeley Symp. Math. Stat. Probab. v. 2. Univ. California Press, Berkeley, Calif., 1972, pp. 583-602.

2. Stein C., Approximation, computation of expectations. Inst. Math. Statist., Hayward, Calif., 1986.

3. Тихомиров А. Н., О скорости сходимости в центральной предельной теореме для слабо зависимых величин. Теория вероятностей и ее применения (1980) 25, №4, 800-818.

4. Форманов Ш. К., Метод Стейна-Тихомирова и неклассическая центральная предельная теорема. Доклады РАН (2001) 376, №4, 458-460.

5. Петровский И. Г. Лекции по теории обыкновенных дифференциальных уравнений. Наука, Москва, 1970.

6. Золотарев В. М., Современная теория суммирования независимых случайных величин. Наука, Москва, 1986.

7. Ротарь В. И., К обобщению теоремы Линдеберга-Феллера. Матем. заметки (1975) 18, №1, $129-135$.

8. Кирьянова Л. В., Ротарь В. И., Оценка скорости сходимости в центральной предельной теореме для мартингалов. Теория вероятностей и ее применения (1991) 36, №, 301-312.

9. Канторович Л. В., Акилов Г. П., Функициональный анализ. Наука, Москва, 1977.

Статья поступила 12.05.2006. 